\title{
Complete genome sequence of a highly divergent astrovirus isolated from a child with acute diarrhea Stacy R Finkbeiner ${ }^{1}$, Carl D Kirkwood ${ }^{2}$ and David Wang*1
}

Address: ${ }^{1}$ Departments of Molecular Microbiology and Pathology \& Immunology, Washington University School of Medicine, St. Louis, MO, USA and ${ }^{2}$ Enteric Virus Research Group, Murdoch Childrens Research Institute, Royal Children's Hospital, Victoria, Australia

Email: Stacy R Finkbeiner - srfinkbe@artsci.wustl.edu; Carl D Kirkwood - carl.kirkwood@mcri.edu.au; David Wang* - davewang@wustl.edu

* Corresponding author

Published: 14 October 2008

Virology Journal 2008, 5:1 17 doi:10.1 186/1743-422X-5-1 I7

This article is available from: http://www.virologyj.com/content/5/I/II7

(C) 2008 Finkbeiner et al; licensee BioMed Central Ltd.

This is an Open Access article distributed under the terms of the Creative Commons Attribution License (http://creativecommons.org/licenses/by/2.0), which permits unrestricted use, distribution, and reproduction in any medium, provided the original work is properly cited.

\begin{abstract}
Background: Astroviruses infect a variety of mammals and birds and are causative agents of diarrhea in humans and other animal hosts. We have previously described the identification of several sequence fragments with limited sequence identity to known astroviruses in a stool specimen obtained from a child with acute diarrhea, suggesting that a novel virus was present.
\end{abstract}

Results: In this study, the complete genome of this novel virus isolate was sequenced and analyzed. The overall genome organization of this virus paralleled that of known astroviruses, with 3 open reading frames identified. Phylogenetic analysis of the ORFs indicated that this virus is highly divergent from all previously described animal and human astroviruses. Molecular features that are highly conserved in human serotypes $\mathrm{I}-8$, such as a $3^{\prime} \mathrm{NTR}$ stem-loop structure and conserved nucleotide motifs present in the $5^{\prime} \mathrm{NTR}$ and ORFIb/2 junction, were either absent or only partially conserved in this novel virus.

Conclusion: Based on the analyses described herein, we propose that this newly discovered virus represents a novel species in the family Astroviridae. It has tentatively been named Astrovirus MLBI.

\section{Background}

Astroviruses are non-enveloped, single stranded, positive sense RNA viruses. Their genomes range from approximately 6 to $8 \mathrm{~kb}$ in length, are polyadenylated, and have both $5^{\prime}$ and $3^{\prime}$ non-translated regions (NTR) [1]. Their genomes have three open reading frames (ORFs) organized from 5' to 3' as follows: ORF $1 \mathrm{a}$, which encodes a serine protease; ORF1b, which encodes the RNA dependent polymerase; and ORF 2, which encodes the structural proteins. A frameshift must occur during the translation of ORF1a in order for ORF1b to be translated. ORF 2 is translated from a sub-genomic RNA and produces a polyprotein which is cleaved by cellular proteases [1].
The family Astroviridae includes 8 closely related human serotypes as well as additional members that infect cattle, sheep, cats, dogs, deer, chickens, turkeys, and ducks [2]. Although some of the animal astroviruses are known to cause hepatitis or nephritis [3], astroviruses typically cause diarrhea in their hosts. Human astrovirus infections most frequently cause watery diarrhea lasting 2-4 days, and less commonly vomiting, headache, fever, abdominal pains, and anorexia in children under the age of 2, the elderly, and immunocompromised individuals [3]. The known human astroviruses account for up to $~ 10 \%$ of sporadic cases of non-bacterial diarrhea in children [4-8]. 
Diarrhea is the third leading infectious cause of death worldwide and is responsible for approximately 2 million deaths each year as well as [9] an estimated 1.4 billion non-fatal episodes $[10,11]$. In children, rotaviruses, caliciviruses, adenoviruses and astroviruses are responsible for the greatest proportion of cases [5,6,12-14]. Most epidemiological studies fail to identify an etiologic agent in $\sim 40 \%$ of diarrhea cases [15-19]. Recently, we conducted viral metagenomic analysis of diarrhea samples using a mass sequencing approach with the explicit goal of identifying novel viruses that may be candidate causes of diarrhea. One of the stool samples we analyzed was collected in 1999 at the Royal Children's Hospital in Melbourne, Australia from a 3-yr old boy with acute diarrhea. Seven sequence reads were identified in this sample that shared $\leq 67 \%$ amino acid identity to known astrovirus proteins, suggesting that a novel astrovirus was present in the sample [20]. In this paper, we report the full sequencing and characterization of the genome of this astrovirus, referred to hereafter as astrovirus MLB1 (AstV-MLB1).

\section{Results and discussion Genome sequencing and analysis}

In the previous metagenomic study [20], we identified seven sequence reads with limited identity to known astroviruses that could be assembled into two small contigs in a clinical stool sample. The contigs had $42-44 \%$, and $59-61 \%$ amino acid identity to human astrovirus serine proteases and RNA-polymerases, respectively. In this study, the complete genome of the astrovirus present in the original stool specimen was sequenced to an average of >3× coverage [GenBank: FJ222451]. The virus has been tentatively named Astrovirus MLB1 (AstV-MLB1). Analysis of the genome showed that AstV-MLB1 has the same genomic organization as other astroviruses. Like other astroviruses, the AstV-MLB1 genome was predicted to encode three open reading frames (ORF1a, ORF1b, and ORF2) and contained both $5^{\prime}$ and $3^{\prime}$ non-translated regions (NTR), as well as a poly-A tail. The complete genome length of AstV-MLB1 was 6,171 bp, excluding the
poly-A tail, slightly shorter when compared to other astrovirus genomes which range in size between $\sim 6,400$ and 7,300 bp [1]. A comparison of AstV-MLB1 genomic elements with those of fully sequenced astroviruses is shown in Table 1.

The ORF 1a of astroviruses encodes a non-structural polyprotein which contains a serine-like protease motif. Pfam analysis revealed a region of ORF1a that has homology to a peptidase domain. In addition, alignment of AstV-MLB1 with other astroviruses revealed that AstV-MLB1 contains the amino acids of the catalytic triad (His, Asp, Ser) which are conserved in the 3C-like protease motif found in other viruses (data not shown) [21]. The residues RTQ which have been suggested to be involved in substrate binding are conserved among the human astroviruses, but vary in other viruses which have the 3C-like motif [21]. In AstVMLB1, the predicted substrate binding residues (ATR) are identical to those found in Ovine astrovirus and not those of the human astroviruses (data not shown).

A second feature of astrovirus ORF1a is the presence of a bipartite nuclear localization signal (NLS) found in human, chicken, and ovine astroviruses, but not turkey astroviruses [22]. A bipartite NLS is characterized as having two regions of basic amino acids separated by a 10 aa spacer. The protein alignment of ORF1a revealed that AstV-MLB1 has a sequence motif similar to the putative NLS of human astroviruses. This region of the genome has also been predicted to potentially encode for a viral genome-linked protein (VPg) [23]. The high sequence similarity observed between AstV-MLB1 and other astroviruses in the motifs identified as essential for a putative VPg suggests that AstV-MLB1 may also encode a VPg (data not shown). While no experimental data exists supporting the prediction of the presence of a Vpg being encoded in any of the astrovirus genomes, we should note that we did encounter difficulty in obtaining the 5 ' end of the MLB1 genome until treatment of the RNA with proteinase $\mathrm{K}$

Table I: Genome Comparison of AstV-MLB I to other astroviruses

\begin{tabular}{|c|c|c|c|c|c|c|}
\hline Virus & Genome (bp) & 5' UTR (bp) & ORFIa & ORFIb & ORF2 & 3' UTR \\
\hline Chicken AstV-I & 6,927 & 15 & 3,017 & 1,533 & 2,052 & 305 \\
\hline Turkey AstV-I & 7,003 & 11 & 3,300 & 1,539 & 2,016 & 130 \\
\hline Turkey AstV-2 & 7,325 & 21 & 3,378 & $\mathrm{I}, 584$ & 2,175 & 196 \\
\hline Mink AstV & 6,610 & 26 & 2,648 & 1,620 & 2,328 & 108 \\
\hline Ovine AstV & 6,440 & 45 & 2,580 & I,572 & 2,289 & 59 \\
\hline Human AstV-I & 6,813 & 85 & 2,763 & $\mathrm{I}, 560$ & $2,36 I$ & 80 \\
\hline Human AstV-2 & 6,828 & 82 & 2,763 & $\mathrm{I}, 560$ & 2,392 & 82 \\
\hline Human AstV-4 & 6,723 & 84 & 2,763 & 1,548 & 2,316 & 81 \\
\hline Human AstV-5 & 6,762 & 83 & 2,763 & I,548 & 2,352 & 86 \\
\hline Human AstV-8 & 6,759 & 80 & 2,766 & $\mathrm{I}, 557$ & 2,349 & 85 \\
\hline AstV-MLB I & $6,|7|$ & 14 & 2,364 & 1,536 & 2,27 I & 58 \\
\hline
\end{tabular}


prior to RNA extraction was added to the experimental protocol.

Finally, the 2,364 nt sequence of AstV-MLB1 ORF1a is shorter than ORF1a sequences of other astroviruses, which range between $\sim 2,500-3,300$ nt (Table 1). The shorter length of AstV-MLB1 ORF1a relative to the human astroviruses is largely attributable to two deletions totaling 57 amino acids located within a highly conserved motif near the carboxyl terminus of human astroviruses $1-8$. This deletion falls within a 144 aa region that has been mapped as being an immunoreactive epitope in human astroviruses [24] and is located in the non-structural protein p38 [21]. Recently, p38 has been reported to lead to apoptosis of the host cell which results in efficient virus replication [25] and particle release [26]. However, it is unclear how the genome deletion identified in AstVMLB1 might influence these activities.

Astrovirus ORF1b is classically generated by a -1 ribosomal frameshift induced by the presence of a heptameric 'slippery sequence' (AAAAAAAC). [2]. A conserved slippery sequence was identified near the end of ORF1a of Ast-MLB1 and FSFinder was used to determine if the downstream sequence was capable of forming a stemloop structure, as found in other astoviruses [27]. The predicted start position of ORF1b was then determined by selecting the first amino acid in frame with the slippery sequence. The $1 \mathrm{~b}$ open reading frame of astroviruses encodes an RNA-dependent RNA polymerase (RNAP). Pfam analysis revealed that AstV-MLB1 ORF1b contains the RNA-dependent RNA polymerase domain found in other positive strand RNA viruses, suggesting this ORF does in fact encode for an RNAP.

Astrovirus ORF2 encodes a large structural polyprotein that is cleaved by cellular proteases to generate the viral capsid proteins. Following the convention of human astroviruses $[28,29]$ by choosing a start codon for ORF2 located two nucleotides upstream of the ORF 1b stop codon resulted in a predicted protein length of 756aa. Pfam analysis of the predicted protein encoded by ORF2 identifies an astrovirus capsid motif, thereby congruent with the paradigm of astrovirus genome organization in which ORF2 encodes the structural capsid proteins.

The AstV-MLB1 ORF2 protein sequence was divided into four subregions for more detailed analysis as described [30]. Pair-wise comparisons of each region were conducted between the AstV-MLB1 sequence and the sequences of all astroviruses for which sequences were available. Consistent with previous reports, region I appeared to be the most conserved of the four regions and in each of the regions, AstV-MLB1 shared the most similarity to known human astroviruses. However, even in region I, AstV-MLB1 only exhibited 33-35\% identity to known human astroviruses. In the less conserved regions II-IV, AstV-MLB1 shared only 5-27\% amino acid identity to the known human astroviruses. By contrast, the range of identities between human astrovirus serotypes 1-8 were, $43-75 \%, 16-66 \%$ and $28-77 \%$ for regions II, III and IV, respectively. Overall, ASTV-MLB1 maintained higher conservation in region I of ORF2 than in other regions, consistent with paradigms established by analysis of other astroviruses.

\section{Non-coding features}

Multiple independent 5' RACE experiments were performed to determine the precise $5^{\prime}$ end of the genome. Based on these experiments, the AstV-MLB1 5' NTR was determined to be $14 \mathrm{nt}$ long. This is similar in length to the 10-20 nt 5'NTRs of avian astroviruses [1], but much shorter than the 80-85 nt long 5'NTRs of the 8 human astrovirus serotypes (Table 1). Notably, the human astroviruses share a $20 \mathrm{nt}$ consensus sequence at the terminal 5 ' nucleotides of the genome which is not conserved in other astroviruses (data not shown). AstV-MLB1 contained 13 out of the 20 consensus nucleotides, including the most 5'CCAA motif within the this region [31] (Fig. 1A). These data support the notion that the sequence we generated does contain the very $5^{\prime}$ terminus of the genome.

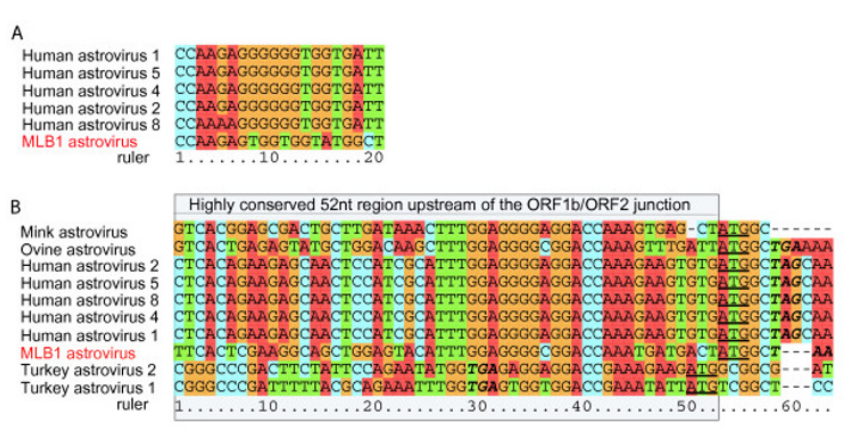

\section{Figure I}

Multiple sequence alignments of putative astrovirus regulatory regions. A.) Alignment of the 20 nucleotides at the very 5 ' end of the Astrovirus MLBI genome with those of fully sequenced astroviruses. MLBI only shares $I 3$ of the 20 conserved nucleotides present in human strains I-8. B.) Alignment of the $52 \mathrm{nt}$ highly conserved nucleotide motif (shown in box) present immediately upstream of the ORFIb/ ORF2 junction of Astrovirus MLBI and other astroviruses. (Note: there is no overlap in the Turkey Astroviruses). MLBI lacks the high degree of sequence identity seen between the human astroviruses. The start codon of ORF2 is shown underlined and the stop codon of ORFIb is shown italicized in bold for each virus. 
Human astroviruses contain a 120 nt region at the junction between ORF1b and ORF2 that is 95-97\% conserved between serotypes [32]. The most highly conserved core $52 \mathrm{nt}$ region of this sequence is $99-100 \%$ identical among the human astrovirus serotypes. The exact role of this sequence is not known, but it is hypothesized to be a regulatory element of the sub-genomic RNA that encodes for ORF2. Alignment between AstV-MLB1 and other human astroviruses of the highly conserved $52 \mathrm{nt}$ at the ORF1b/ORF2 junction revealed that AstV-MLB1 possessed only $61.5 \%$ identity in this region (Fig. 1B). By contrast, the known animal astroviruses share only $44-59.6 \%$ identity in this $52 \mathrm{nt}$ region with human astroviruses as determined by pair-wise comparisons. Interestingly, AstVMLB1 shares $71.2 \%$ identity in this region to Ovine Astrovirus.

All of the previously described astroviruses, with the exception of turkey astrovirus 2, have a conserved RNA secondary structure referred to as the stem-loop II-like motif ( $\mathrm{s} 2 \mathrm{~m}$ ) found at the $3^{\prime}$ end of the genome in the $3^{\prime}$ NTR [33]. This motif is also present in some coronaviruses and equine rhinovirus serotype 2 . Mutations within this motif are generally accompanied by compensatory mutations that restore base pairing [33]. The conservation of such a sequence motif across multiple viral families suggests that it may play a broad role in the biology of positive stranded RNA viruses [33]. The exact function of this stem loop is not known, but it is hypothesized to interact with viral and cellular proteins needed for RNA replication. Nucleotide alignment of the 150 nucleotides at the 3 ' terminus of the AstV-MLB1 genome and other viruses known to contain the stem-loop motif suggested that AstV-MLB1 does not have this conserved nucleotide motif (data not shown). Furthermore, it also has the shortest 3'NTR reported to date for an astrovirus. (Table 1) [1].

\section{Phylogenetic analysis}

Multiple sequence alignments of the three astrovirus open reading frames were performed and bootstrapped maximum parsimony trees were generated (Fig. 2). The trees confirmed initial assessments that AstV-MLB1 is a novel astrovirus[20]. The trees for ORFs $1 \mathrm{a}$ and $1 \mathrm{~b}$ (Fig. 2a, b) both indicated that AstV-MLB1 is most closely related to the human astroviruses, although it is highly divergent from them. AstV-MLB1 ORF1a only has 9-28\% amino acid identity to other astrovirus ORF1a proteins and the pairwise sequence alignments of ORF1b revealed 35-54\% amino acid identity between ORF1b proteins of AstVMLB1 and other astroviruses (Table 2). The maximum parsimony tree for ORF2 (Fig. 2c) shows that there is greater divergence among all of the sequences for ORF2, as is to be expected of the capsid region. However it is still evident that AstV-MLB1 is quite divergent from any of the known human astroviruses. Based on the predicted $756 \mathrm{aa}$ protein of ORF2, AstV-MLB1 has only $11-24 \%$ amino acid identity to other astrovirus capsid precursor proteins (Table 2).

\section{Origin of virus}

At this point, the origin of AstV-MLB1 is unclear. AstVMLB1 may be a bona fide human virus capable of infecting and replicating within the human gastrointestinal tract that had evaded detection until now. Alternately, it may be a passenger virus present simply as a result of dietary ingestion, as has been described previously for plant viruses detected in human stool [34]. Of course, viruses derived from dietary intake that appear to cause human disease, such as Aichi virus, have been described previously $[35,36]$. Another possibility is that this virus may represent zoonotic transmission from some other animal species that is the true host for Astrovirus MLB1. Traditionally it has been thought that astroviruses have a strict species tropism. However, recent evidence has emerged that suggests that interspecies transmission does occur. For example, chicken astrovirus antibodies have been detected in turkeys [37] and an astrovirus was isolated from humans whose capsid sequence most closely resembled that of feline astrovirus[1]. Because of the uncertainty as to the identity of the true host species and the host range for this virus, we have tentatively named this novel virus Astrovirus MLB1 (AstV-MLB1). Efforts to define whether AstV-MLB1 is a novel human pathogen are underway.

\section{Conclusion}

Complete sequencing and genome analysis of Astrovirus MLB1 revealed that the virus has three open reading

Table 2: Comparison of astrovirus proteins to predicted AstV-MLB I proteins

\begin{tabular}{|c|c|c|c|c|c|c|c|c|c|c|c|c|c|c|c|}
\hline \multirow[t]{2}{*}{ ORF } & \multirow{2}{*}{$\begin{array}{l}\text { Est. } \\
\text { Size } \\
\text { (aa) }\end{array}$} & \multicolumn{14}{|c|}{ \% Amino Acid Identity to: } \\
\hline & & $\begin{array}{c}\text { HAstV } \\
-1\end{array}$ & $\begin{array}{c}\text { HAstV } \\
-2\end{array}$ & $\begin{array}{c}\text { HAstV } \\
-3\end{array}$ & $\begin{array}{c}\text { HAstV } \\
-4\end{array}$ & $\begin{array}{c}\text { HAstV } \\
-5\end{array}$ & $\begin{array}{c}\text { HAstV } \\
-6\end{array}$ & $\begin{array}{c}\text { HAstV } \\
-7\end{array}$ & $\begin{array}{c}\text { HAstV } \\
-8\end{array}$ & $\begin{array}{c}\text { TAstV } \\
-1\end{array}$ & $\begin{array}{c}\text { TAstV } \\
-2\end{array}$ & $\begin{array}{c}\text { TAstV } \\
-3\end{array}$ & $\begin{array}{c}\text { ChAst } \\
\text { V-I }\end{array}$ & OAstV & MAstV \\
\hline Ia & 787 & 28 & 28 & NA & 29 & 29 & NA & NA & 29 & 9 & 9 & NA & 10 & 22 & 24 \\
\hline $\mathrm{Ib}$ & 511 & 54 & 54 & NA & 54 & 54 & NA & NA & 54 & 36 & 35 & NA & 36 & 47 & 44 \\
\hline 2 & 756 & 24 & 24 & 24 & 23 & 23 & 24 & 24 & 24 & 15 & 16 & 16 & II & 18 & 19 \\
\hline
\end{tabular}



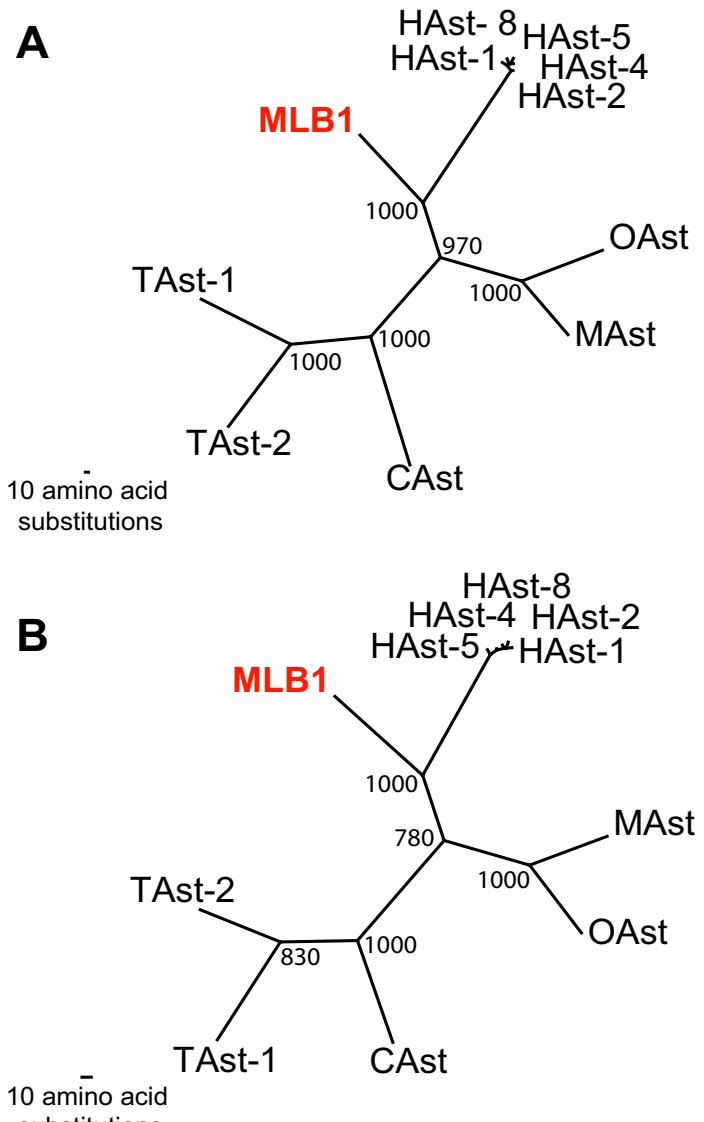

substitutions

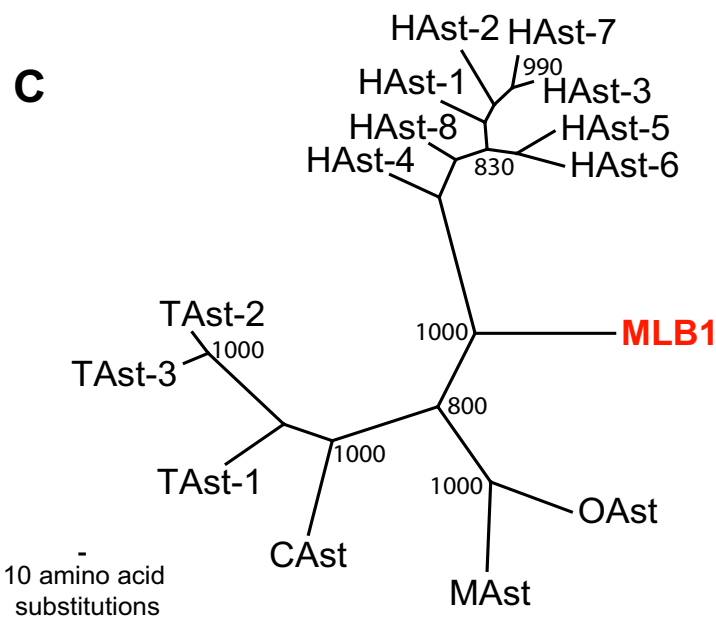

Figure 2

Phylogenetic analysis of AstV-MLB I open reading frames. Phylogenetic trees are based on amino acid sequences and were generated using the maximum parsimony method with I,000 bootstrap replicates. Significant bootstrap values are shown. (A) ORFIa; (B) ORFIb; (C) ORF2. HAstV = Human astrovirus; $\mathrm{CAstV}=$ Chicken astrovirus; $\mathrm{MAstV}=$ Mink astrovirus; $\mathrm{TAstV}=$ Turkey astrovirus; OAst $\mathrm{V}=$ Ovine astrovirus. frames sharing the same organization as other astroviruses. Phylogenetic analysis of the open reading frames clearly demonstrated that AstV-MLB1 is highly divergent from any of the known astroviruses. Furthermore, AstVMLB1 lacks the conservation seen between human astroviruses $1-8$ in the non-translated regions of the genome such as the $5^{\prime}$ and 3 ' NTR and the ORF1b/2 junction. The aggregate analysis of the non-coding features and ORFs as well as the phylogentic analysis clearly indicates that AstVMLB1 is highly divergent from all previously described astroviruses.

The divergence of AstV-MLB1 from known astroviruses in the non-translated regions of the genome is particularly interesting because these regions are nucleotide motifs that are thought to play regulatory roles in viral replication. This suggests that AstV-MLB1 may behave very differently from the known astroviruses and that additional studies on the regulation of AstV-MLB1 transcription and replication may broaden our understanding of astrovirus paradigms.

Astroviruses are associated with diarrhea predominantly in young children and immunocompromised individuals. The discovery of AstV-MLB1 in a liver transplant patient fits well with the known clinical parameters of astrovirus infection. We previously reported that the only other virus detected in this stool was a TT virus [20], which is thought to be non-pathogenic [38]. It is therefore tempting to speculate that AstV-MLB1 is the pathogenic agent that caused this case of diarrhea. However, whether AstVMLB1 is a bona fide human virus capable of causing diarrhea will have to be established by further experimentation and epidemiological surveys.

\section{Methods \\ Specimen}

A stool sample was collected from a 3 year old boy admitted to the Royal Children's Hospital with acute diarrhea in 1999. The child had previously undergone a liver transplant one year prior to this episode of diarrhea, however the immunological status was unknown.

\section{RNA extraction}

RNA was isolated from the primary stool filtrate using RNA-Bee (Tel-Test, Inc.) according to manufacturer's instructions. In some cases, the stool filtrate was treated with $2.5 \mathrm{mg} \backslash \mathrm{ml}$ proteinase K (Sigma) for $30 \mathrm{~min}$ prior to RNA extraction.

\section{Genome amplification and sequencing}

The astrovirus sequence reads previously detected in the primary stool filtrate [20] [GenBank accessions: ET065575, ET065576, ET065577, ET065579, ET065580, ET065581, ET065582] were assembled into two contigs, 
and the nucleic acid between the contigs was obtained by RT-PCR. For reverse transcription reactions, cDNA was generated with MonsterScript RT at $65^{\circ} \mathrm{C}$ and amplified with Taq (Invitrogen). Subsequent $5^{\prime}$ and $3^{\prime}$ RACE reactions were done to obtain the entire genome. To generate high quality sequence coverage, 7 pairs of specific primers that spanned the complete genome in overlapping $\sim 1 \mathrm{~kb}$ fragments were used in RT-PCR reactions and then cloned and sequenced using standard Sanger sequencing chemistry. All amplicons were cloned into pCR4.0 (Invitrogen). These 7 primer pairs were used to confirm the sequence of the viral genome from both the primary stool sample and the passage 2 tissue culture sample. The complete genome sequence of AstV-MLB1 has been deposited in [GenBank: FJ222451].

\section{ORF prediction and annotation}

Open reading frames $1 \mathrm{a}$ and 2 were predicted for AstVMLB1 using the NCBI ORF Finder program. ORF1b was predicted based on the frameshift paradigm that occurs in other astroviruses by identifying a heptameric slippery sequence [39]. Conserved motifs were identified using Pfam [40].

\section{Pair-wise alignments}

Bioedit was used to determine the percent identity between sequences as determined by pair-wise alignments.

\section{Phylogenetic analysis}

ClustalX (1.83) was used to carry out multiple sequence alignments of the protein sequences associated with all three of the open reading frames of representative astrovirus types. Maximum parsimony trees were generated using PAUP with 1,000 bootstrap replicates [41]. Available nucleotide or protein sequences of the following astroviruses were obtained: Human Astrovirus 1 [GenBank: NC 001943]; Human Astrovirus 2 [GenBank: L13745]; Human Astrovirus 3 [GenBank: AAD17224]; Human Astrovirus 4 [GenBank: DQ070852]; Human Astrovirus 5 [GenBank: DQ028633]; Human Astrovirus 6 [EMBL: CAA86616]; Human Astrovirus 7 [Gen Bank: AAK31913]; Human Astrovirus 8 [GenBank: AF260508]; Turkey Astrovirus 1 [GenBank: Y15936]; Turkey Astrovirus 2 [GenBank: NC_005790]; Turkey Astrovirus 3 [GenBank: AY769616]; Chicken Astrovirus [GenBank: NC 003790]; Ovine Astrovirus [GenBank: NC 002469]; and Mink Astrovirus [GenBank: NC 004579].

\section{Competing interests}

The authors declare that they have no competing interests.

\section{Authors' contributions}

DW conceived and designed the experiments. SF carried out the experiments and analysis. $\mathrm{CK}$ contributed reagents/materials. SF and DW wrote the paper.

\section{Acknowledgements}

This work was funded in part by an NHMRC RD Wright Research Fellowship (ID 334364, CK), and by the Food Safety Research Response Network, a Coordinated Agricultural Project, funded through the National Research Initiative of the USDA Cooperative State Research, Education and Extension Service, grant number \#2005-352 I2-15287.

\section{References}

I. Mendez E, Arias CF: Astroviruses. In Fields Virology Volume I. 5th edition. Edited by: Knipe DM, Howley PM. Philadelphia: Lippincott WIIlliams \& Wilkins; 2007:98I-1000.

2. Koci MD, Schultz-Cherry S: Avian astroviruses. Avian Pathol 2002, 3 I:2 I 3-227.

3. Moser LA, Schultz-Cherry S: Pathogenesis of astrovirus infection. Viral Immunol 2005, I 8:4-10.

4. Glass RI, Noel J, Mitchell D, Herrmann JE, Blacklow NR, Pickering LK, Dennehy P, Ruiz-Palacios G, de Guerrero ML, Monroe SS: The changing epidemiology of astrovirus-associated gastroenteritis: a review. Arch Virol Suppl 1996, I 2:287-300.

5. Klein EJ, Boster DR, Stapp JR, Wells JG, Qin X, Clausen CR, Swerdlow DL, Braden CR, Tarr PI: Diarrhea Etiology in a Children's Hospital Emergency Department: A Prospective Cohort Study. Clin Infect Dis 2006, 43:807-8I3.

6. Kirkwood CD, Clark R, Bogdanovic-Sakran N, Bishop RF: A 5-year study of the prevalence and genetic diversity of human caliciviruses associated with sporadic cases of acute gastroenteritis in young children admitted to hospital in Melbourne, Australia (1 998-2002). J Med Virol 2005, 77:96-I0I.

7. Soares CC, Maciel de Albuquerque MC, Maranhao AG, Rocha LN, Ramirez ML, Benati FJ, Timenetsky Mdo C, Santos N: Astrovirus detection in sporadic cases of diarrhea among hospitalized and non-hospitalized children in Rio De Janeiro, Brazil, from 1998 to 2004. J Med Virol 2008, 80: I I3-II7.

8. Caracciolo S, Minini C, Colombrita D, Foresti I, Avolio M, Tosti G, Fiorentini S, Caruso A: Detection of sporadic cases of Norovirus infection in hospitalized children in Italy. New Microbiol 2007, 30:49-52.

9. World Health Report. World Health Organization; 2004.

10. O'Ryan M, Prado V, Pickering LK: A millennium update on pediatric diarrheal illness in the developing world. Semin Pediatr Infect Dis 2005, I 6:125-136.

II. Kosek M, Bern C, Guerrant RL: The global burden of diarrhoeal disease, as estimated from studies published between $\mathbf{1 9 9 2}$ and 2000. Bulletin of the World Health Organization 2003, 81 : I 97-204.

12. Nataro JP, Mai V, Johnson J, Blackwelder WC, Heimer R, Tirrell S, Edberg SC, Braden CR, Glenn Morris J Jr, Hirshon JM: Diarrheagenic Escherichia coli infection in Baltimore, Maryland, and New Haven, Connecticut. Clin Infect Dis 2006, 43:402-407.

13. Clark $B$, McKendrick $M$ : $\mathbf{A}$ review of viral gastroenteritis. Curr Opin Infect Dis 2004, I 7:46I-469.

14. Wilhelmi I, Roman E, Sanchez-Fauquier A: Viruses causing gastroenteritis. Clin Microbiol Infect 2003, 9:247-262.

15. Davidson G, Townley R, Bishop RF, Holmes I, Ruck B: Importance of a new virus in acute sporadic enteritis in children. The Lancet 1975:242-246.

16. Kapikan A: Viral Gastroenteritis. The Journal of the American Medical Association 1993, 269:627-630.

17. Kurtz JB, Lee TW, Craig JW, Reed SE: Astrovirus infection in volunteers. I Med Virol 1979, 3:221-230.

18. Thornhill T, Kalica A, Wyatt R, Kapikan A, Chanock R: Pattern of Shedding of the Norwalk Particle in Stools during Experimentally Induced Gastroenteritis in Volunteers as Determined by Immune Electron Microscopy. The Journal of Infectious Diseases 1975, I32:28-34.

19. Wigand R, Baumeister H, Maass G, Kuhn J, Hammer H: Isolation and Identification of Enteric Adenoviruses. Journal of Medical Virology 1983, I I:233-240.

20. Finkbeiner SR, Allred AF, Tarr PI, Klein EJ, Kirkwood CD, Wang D: Metagenomic analysis of human diarrhea: viral detection and discovery. PLoS Pathog 2008, 4: el 0000II.

21. Kiang D, Matsui SM: Proteolytic processing of a human astrovirus nonstructural protein. J Gen Virol 2002, 83:25-34.

22. Jonassen CM, Jonassen TT, Sveen TM, Grinde B: Complete genomic sequences of astroviruses from sheep and turkey: comparison with related viruses. Virus Res 2003, 91:195-201. 
23. Al-Mutairy B, Walter JE, Pothen A, Mitchell DK: Genome Prediction of Putative Genome-Linked Viral Protein (VPg) of Astroviruses. Virus Genes 2005, 3 I:2I-30.

24. Matsui SM, Kim JP, Greenberg HB, Young LM, Smith LS, Lewis TL, Herrmann JE, Blacklow NR, Dupuis K, Reyes GR: Cloning and characterization of human astrovirus immunoreactive epitopes. J Virol 1993, 67: 17|2-17|5.

25. Guix S, Bosch A, Ribes E, Dora Martinez L, Pinto RM: Apoptosis in astrovirus-infected CaCo-2 cells. Virology 2004, 3 19:249-26I.

26. Mendez E, Salas-Ocampo E, Arias CF: Caspases mediate processing of the capsid precursor and cell release of human astroviruses. J Virol 2004, 78:860I-8608.

27. Moon S, Byun Y, Kim HJ, Jeong S, Han K: Predicting genes expressed via $-I$ and $+I$ frameshifts. Nucleic Acids Res 2004, 32:4884-4892.

28. Monroe SS, Jiang B, Stine SE, Koopmans M, Glass RI: Subgenomic RNA sequence of human astrovirus supports classification of Astroviridae as a new family of RNA viruses. J Virol 1993, 67:36II-36I4.

29. Willcocks MM, Carter MJ: Identification and sequence determination of the capsid protein gene of human astrovirus serotype I. FEMS Microbiol Lett 1993, I I 4:I-7.

30. Wang OH, Kakizawa J, Wen LY, Shimizu M, Nishio O, Fang ZY, Ushijima $\mathrm{H}$ : Genetic analysis of the capsid region of astroviruses. J Med Virol 200I, 64:245-255.

31. Mendez-Toss M, Romero-Guido P, Munguia ME, Mendez E, Arias CF: Molecular analysis of a serotype 8 human astrovirus genome. J Gen Virol 2000, 81:289|-2897.

32. Walter JE, Briggs J, Guerrero ML, Matson DO, Pickering LK, Ruiz-Palacios G, Berke T, Mitchell DK: Molecular characterization of a novel recombinant strain of human astrovirus associated with gastroenteritis in children. Arch Virol 200I, 146:2357-2367.

33. Monceyron C, Grinde B, Jonassen TO: Molecular characterisation of the 3'-end of the astrovirus genome. Arch Virol 1997 1 42:699-706.

34. Zhang T, Breitbart M, Lee WH, Run JQ, Wei CL, Soh SW, Hibberd ML, Liu ET, Rohwer F, Ruan Y: RNA viral community in human feces: prevalence of plant pathogenic viruses. PLOS Biol 2006, 4:e3.

35. Yamashita T, Sakae K, Ishihara Y, Isomura S, Utagawa E: Prevalence of newly isolated, cytopathic small round virus (Aichi strain) in Japan. J Clin Microbiol 1993, 3 I:2938-2943.

36. Yamashita T, Kobayashi S, Sakae K, Nakata S, Chiba S, Ishihara Y, Isomura S: Isolation of cytopathic small round viruses with BS-CI cells from patients with gastroenteritis. I Infect Dis |99|, 164:954-957.

37. Baxendale $\mathrm{W}$, Mebatsion $\mathrm{T}$ : The isolation and characterisation of astroviruses from chickens. Avian Pathol 2004, 33:364-370.

38. Bendinelli M, Pistello M, Maggi F, Fornai C, Freer G, Vatteroni ML: Molecular properties, biology, and clinical implications of TT virus, a recently identified widespread infectious agent of humans. Clin Microbiol Rev 200I, I 4:98-II3.

39. Jiang B, Monroe SS, Koonin EV, Stine SE, Glass RI: RNA sequence of astrovirus: distinctive genomic organization and a putative retrovirus-like ribosomal frameshifting signal that directs the viral replicase synthesis. Proc Natl Acad Sci USA 1993 90:10539-10543.

40. Finn RD, Mistry J, Schuster-Bockler B, Griffiths-Jones S, Hollich V, Lassmann T, Moxon S, Marshall M, Khanna A, Durbin R, et al.: Pfam: clans, web tools and services. Nucleic Acids Res 2006, 34:D247-25I.

41. Swofford DL: PAUP*. Phylogenetic Analysis Using Parsimony (*and Other Methods). Version 4th edition. Sunderland, Massachusettes: Sinauer Associates; 1998.
Publish with Bio Med Central and every scientist can read your work free of charge

"BioMed Central will be the most significant development for disseminating the results of biomedical research in our lifetime. "

Sir Paul Nurse, Cancer Research UK

Your research papers will be:

- available free of charge to the entire biomedical community

- peer reviewed and published immediately upon acceptance

- cited in PubMed and archived on PubMed Central

- yours - you keep the copyright

Submit your manuscript here:

http://www.biomedcentral.com/info/publishing_adv.asp
BioMedcentral 